\title{
Performance of various colored sticky traps in monitoring against insects of maize crop
}

\author{
Asad Rajput ${ }^{1 *}$, Ahmed Lund Baloch ${ }^{1}$, Junaid Ahmed Soomro ${ }^{1}$ and \\ Ghulam Qadeer Junejo ${ }^{1}$
}

1. Department of Entomology, Sindh Agriculture University Tandojam-Pakistan

*Corresponding author's email: iamasadrajput@gmail.com

Citation

Asad Rajput, Ahmed Lund Baloch, Junaid Ahmed Soomro and Ghulam Qadeer Junejo. Performance of various colored sticky traps in monitoring against insects of maize crop. Pure and Applied Biology. Vol. 10, Issue 2, pp348359. http://dx.doi.org/10.19045/bspab.2021.100038

\begin{tabular}{llll}
\hline \hline Received: 08/05/2020 & Revised: 05/08/2020 & Accepted: 26/08/2020 & Online First: 28/09/2020 \\
\hline \hline
\end{tabular}

\section{Abstract}

The field study on relative performance of various colored sticky traps against insects of maize crop (Zea mays L.) was conducted during 2019. Five different colored sticky traps i.e., yellow, blue, green, white and transparent were installed, where each trap was replicated four times. The field was divided into four blocks, each having a size of 30,000 square feet. In each block, five colored sticky traps were randomly installed at one feet above the crop height. Weekly observations were taken to count the number of insects attracted to various colored from germination till harvesting of crop. Identification of insects were done on available literature. Data of abiotic factors were also obtained to determine their influence on attractiveness of colored sticky traps for insects. During the study, five insect pests and one predator species were found i.e., Rhospalosipum maidis, Cicadulina mbila, Frankliniella occidentali, Atherigona soccata, Adelphocoris suturalis and Cheilomenes sexmaculata on various colored traps. All the insects were strongly attracted toward yellow colored traps as compere to others. The overall weekly observation showed that peak populations of $R$. maidis, $F$. occidentali, A. suturalis, $C$. sexmaculata were recorded on $31^{\text {st }}$ March, 2019 on various colored traps. The maximum population of $C$. mbila and A. soccata was recorded on $6^{\text {th }}$ April and $5^{\text {th }}$ May, respectively. The populations of $R$. maidis, Cicadulina mbila, $F$. occidentali and $C$. sexmaculata showed positive and significant. Weakly a-biotic factors significantly affected on the performance of colored sticky traps to attract various insect. Based on the findings, yellow colored traps should be used for the early detection and monitoring of maize insects.

Key words: Maize insects; performance; Sticky traps

\section{Introduction}

Maize (Zea mays L.) (Family Poaceae) is a yearly, cross-pollinated, kharif crop. It provides nourishment to people, feed for animals and raw material for many important agro-based industries [1]. It is estimated that maize crop will be the largest staple crop of developing world by 2050 and the demand for maize in the developing world is expected to double [2]. Maize is third and largest growing crop after wheat and rice and the USA is the largest maize producing country [3]. In Pakistan, maize ranked as fourth largest cultivated crop after wheat, cotton and rice. Except for potato, maize crop is the most gainful, stable and reliable crop in Pakistan [4].

Although, the maize is grown on large scale in Pakistan, but its per acre yield is comparatively very low than globe. The 
lower yield of maize is attributed to various biotic and a-biotic factors [5]. In Pakistan 2016-2017, the total production of maize was recorded at 6.134 million tons with area under cultivation of 1348,000 hectors areas, however, both production and area under cultivation declined during 2017-18 and remained at 5.702 million tons and 1229,000 hectares, respectively, showing a decline of $7.0 \%$ [6] (GOP, 2018). There are numerous elements which are responsible for the low yield of maize within which insect pests are major ones [7].

Several control measures are applied to control the infestation and to increase per acre grain as well as green fodder yield. Generally, the maize growers control the pests through the application of chemical insecticides [8]. The use of chemical insecticides is highly practiced particularly in developing countries including Pakistan. Chemicals can suppress the pest population rapidly below the threshold, but their continuous uses introduce many environmental problems [9]. Therefore, it is urgent need to introduce eco-friendly control measures against insect pests. Plantation of resistant varieties is the best approach to overcoming the problems of synthetic insecticides [10]. Though the chewing insect is major pest status, the control of this pest through nonchemical practices (biological, cultural, and host plant resistance etc.) utilized by the advance farmers and researchers throughout the world are insufficient [11]. Thus, an effective population scouting and monitoring is crucial for successful implementation of insect and vector control programs [12]. Among the monitoring tools, use of sticky colored traps are one of the effective tools for insect pests scouting in agro-ecosystem. The use of sticky traps gives a basic technique for monitoring relative evaluations of pest population with small efforts mostly used in integrated pest management programs in diverse agricultural crops [13]. Recently, various colored sticky traps are used to determine the presence and monitoring of many important insect pests of various crops such as thrips, white flies, hoppers etc. [14]. The attraction of pest towards trap crop is based on the plantation time along with spacing of trap crop and the main crop. Therefore, considering the importance of sticky traps in early detection and monitoring of insect pests, this study was designed to evaluate the performance of different colored sticky traps against insects of maize.

\section{Materials and Methods}

\section{Experimental setup, data recording and analysis}

The maize variety P1429 was sown at its recommended seed rate $(9.5 \mathrm{~kg} / \mathrm{acre})$ at a farmer's field located at Palejani Village, Taluka and District Matiari. The distance between row to row and plant to plant was managed at 27 inches and 7 inches, respectively. The experiment was arranged in a Randomized Complete Block Design (RCBD) as farmer's field was divided into four blocks, each block with a size of $30 \times 100$ feet. Thus, the total experimental area used in the study was 120,000 sq. feet. In each block, five colored sticky traps i.e., white, yellow, green, blue and transparent were randomly installed at one feet above the crop height. Thus, height of the traps were adjusted according to the crop stage. The size of individual trap was $1 \times 1$ square feet. The sticky traps were glued with transparent lubricant from both size to maximize the number of insects' attraction.

Observations on insects attracted to various colored sticky traps were recorded on weekly basis from germination till harvesting of crop. After each observation, sticky traps were thoroughly washed using detergent and re-glued as mentioned above. The data of temperature, relative humidity and wind velocity was obtained from the 
Meteorological Station, TandoJam to determine their influence on the attractiveness of colored sticky traps for insects. Identification of insects were done on available literature. [15-18]

After data collection, Analysis of Variance was used to analysis the data, whereas, the Least Square Difference test was applied to determine the means with significant differences. Moreover, Pearson's correlation was applied to get the relationship of insect's attraction on various colored sticky traps with respect to temperature, relative humidity and wind velocity. The Statistix 8.1 computer software was use $\mathrm{d}$ for the entire analysis.

\section{Results}

The present study was conducted to evaluate the performance of sticky traps of five different colored (white, yellow, green, blue and transparent) to attract various insects of maize crop under field conditions. During the study six different species of insects attracted toward the sticky traps. Namely aphids (Rhospalosipum maidis), mirid bugs, (Adelphocoris suturalis) leafhopper, (Cicadulina mbila), shootfly (Atherigona soccata), thrip (Frankliniella occidentali), zigzag beetle (Cheilomenes sexmaculata) was recorded. The detailed results are discussed below:

The result of the effects of various colored sticky traps on attractiveness of aphids (Rhospalosipum maidis) is shown in (Table 1 ) indicated that the highest population of $R$. maidis (56.23 \pm 16.18$)$ was recorded on yellow sticky traps, followed by blue $(33.68 \pm 10.00)$, green $(29.45 \pm 8.64)$, white $(15.84 \pm 3.89)$ and transparent $(9.70 \pm 2.25)$ colored traps. Thus, overall the maximum number of $R$. maidis attracted to various sticky traps was recorded on $31^{\text {st }}$ March $(101.90 \pm 31.42)$, followed by $6^{\text {th }}$ April (65.40 \pm 20.48$)$, whereas, the minimum number of $R$. maidis $(0.55 \pm 0.39)$ was recorded on $16^{\text {th }}$ March, followed by $27^{\text {th }}$
April (1.75 \pm 1.08$)$. The ANOVA results indicated in Appendix I that highly significant difference $(\mathrm{F}=108.90, \mathrm{P}<$ $0.001)$ of $R$. maidis population was recorded on various colored traps. Whereas Results also showed that $R$. maidis population showed a significant difference $(\mathrm{F}=108.26$, $\mathrm{P}<0.001)$ on various observation dates.

The (Table 2) showed the results regarding the attractiveness of leafhoppers (Cicadulina mbila) on different colored sticky traps that showed almost the similar trend as that of $R$. maidis. Accordingly, the maximum attraction of $C$. mbila $(43.02 \pm 15.91)$ was recorded on yellow sticky traps, whereas, the minimum population was observed $(23.14 \pm 8.82)$ on white sticky traps. Thus, the highest number of $C$. mbila was attracted on $6^{\text {th }}$ April $(130 \pm 14.71)$, followed by $31^{\text {st }}$ March (81.75 \pm 14.22$)$. Whereas, the minimum number of $C$. mbila were recorded $(4.85 \pm 1.13)$ on $16^{\text {th }}$ May, followed by $27^{\text {th }}$ May (5.44 \pm 1.43$)$. Thus, a significant difference $(\mathrm{F}=4.92, \mathrm{P}<0.001)$ was recorded on the level of attraction of different colored sticky traps to attract $C$. mbila. Results also showed in Appendix II that highly significant difference $(\mathrm{F}=39.99$, $\mathrm{P}<0.0001)$ was also recorded for number of C. mbila attracted to different colored sticky traps on various observation dates.

The (Table 3 ) indicated that the population of $F$. occidentali was started in first week of March, increased till last week of March and then started decreasing. Peak population of F. occidentali (122.05 \pm 3.06$)$ was recorded on $31^{\text {st }}$ March, followed by $6^{\text {th }}$ April (61.25 \pm 9.76$)$, whereas the lowest F. occidentali population $(8.95 \pm 2.40)$ was recorded on $9^{\text {th }}$ May, followed by (14.3 \pm 1.36$) 2^{\text {nd }}$ May and then completely disappeared afterwards till last week of May. According to sticky traps result also showed the maximum population of F. occidentali $(31.08 \pm 13.81)$ was recorded on yellow sticky traps, Followed by white 
$(22.41 \pm 12.32)$,

blue (20.64 \pm 8.91$)$,

transparent

(18.14 \pm 8.63$), \quad$ and green

$(11.78 \pm 3.23)$ color traps. The ANOVA results illustrated in Appendix III that the attractiveness of thrips (Frankliniella occidentali) population varied highly significantly $(\mathrm{F}=42.15, \mathrm{P}<0.001)$ throughout various observation dates on different colored sticky traps. According to sticky traps result also showed that the highly significant difference $(\mathrm{F}=5.51, \mathrm{P}<$ 0.001 ) of $F$. occidentali population was recorded on various colored traps.

The ANOVA results of shoofly (Atherigona soccata) showed in Appendix IV that the highly significant difference $(\mathrm{F}=15.69, \mathrm{P}<$ 0.001 ) regarding their attraction on various colored sticky traps. ANOVA results also indicated highly significant difference $(\mathrm{F}=$ 20.06, $\mathrm{P}<0.001$ ) among population of $A$. soccata on various observation dates. Whereas, the (Table 4) Indicated that the maximum population of $A$. soccata $(3.35 \pm 1.09)$ was recorded on yellow sticky traps, followed by green $(1.35 \pm 0.60)$, blue $(1.26 \pm 0.48)$, transparent $(0.78 \pm 0.26)$ and white $(0.75 \pm 0.28)$ colored traps. The $A$. soccata population gradually started during $1^{\text {st }}$ week of March to last week of March. Then, population of $A$. soccata was not recorded up to last week of April. Then $A$. soccata population increased during $2^{\text {nd }}$ week of May and reached its peak $(6.65 \pm 1.76)$ on the $1^{\text {st }}$ week of May. Whereas, the lowest population $(0.45 \pm 0.2)$ was recorded on $22^{\text {nd }}$ March. Then, again $A$. soccata population disappeared continually till harvesting of the crop.

The result of the effects of various colored sticky traps on attractiveness of mirid bugs (Adelphocoris suturalis) are shown in (Table 5) The highest numbers of $A$. suturalis was recorded $(1.35 \pm 0.28)$ on $31^{\text {st }}$ March, followed by $8^{\text {th }}$ March $(0.95 \pm 0.22)$. Thus, the minimum numbers of $A$. suturalis were recorded $(0.25 \pm 0.16)$ on $15^{\text {th }}$ March, followed by $16^{\text {th }} 27^{\text {th }}$ May $(0.30 \pm 0.15)$. The maximum population of $A$. suturalis $(1.06 \pm 0.18)$ was recorded on yellow sticky traps, followed by green $(0.60 \pm 0.12)$, white $(0.42 \pm 0.18)$, transparent $(0.33 \pm 0.12)$ and blue $(0.33 \pm 0.11)$ color traps. Whereas, the ANOVA results indicated in Appendix $\mathrm{V}$ that $A$. suturalis population showed highly significant difference $(\mathrm{F}=5.46, \mathrm{P}<0.001)$ on various observation dates. Result also showed that highly significant difference $(\mathrm{F}$ $=8.24, \mathrm{P}<0.001)$ of A suturalis population was recorded on various colored traps.

In (Table 6) showed the results regarding the attractiveness of zigzag beetles (Cheilomenes sexmaculata) on various colored sticky traps that showed almost the similar trend as that of C. mbila. Accordingly, the peak population of $C$. sexmaculata $(1.5 \pm 0.26)$ was recorded on yellow sticky traps, whereas the minimum population $(0.5 \pm 0.10)$ was recoded on white colored traps. According to weekly observation dates, the highest number of $C$. sexmaculata was recorded on $31^{\text {st }}$ March $(1.65 \pm 0.25), \quad$ followed by, $2^{\text {nd }}$ May (1.45 \pm 0.59$)$. Moreover, the minimum number of $C$. sexmaculata were recorded $(0.40 \pm 0.20)$ on $20^{\text {th }}$ April, followed by $16^{\text {th }}$ April $27^{\text {th }}$ May $(0.55 \pm 0.15)$. Thus, a significant difference $(\mathrm{F}=9.01, \mathrm{P}<0.001)$ was recorded on the level of attraction of different colored sticky trap showed in Appendix VI. ANOVA results also indicated that $C$. sexmaculata population showed a highly significant difference $(\mathrm{F}=4.76, \mathrm{P}<$ 0.001 ) on various observation dates.

\section{Pearson's correlation between insect} populations and a-biotic factors

The correlation results given in (Table 7) indicated that temperature showed a positive, significant but weak influence on the population of $R$. maidis $(\mathrm{r}=0.1314, \mathrm{P}<$ 0.05), C. mbila $(\mathrm{r}=0.1921, \mathrm{P}<0.05), F$. occidentali $(\mathrm{r}=0.1287, \mathrm{p}<0.05)$ and $C$. sexmaculata $(\mathrm{r}=0.1207, \mathrm{p}=<0.05)$, 
whereas, no significant impact of temperature was recorded on the population of A. soccata $(\mathrm{r}=0.0420, \mathrm{p}=>0.05)$ and $A$. suturalis $(\mathrm{r}=0.1003, \mathrm{p}=>0.05)$. Moreover, relative humidity showed a significant, weak but negative relationship with population of $R$. maidis $(\mathrm{r}=-0.3061, \mathrm{p}=<0.05), C$. mbila ( $\mathrm{r}=-0.1572=, \mathrm{p}=<0.05)$ and $F$. occidentali $(\mathrm{r}=-0.2572=, \mathrm{p}=<0.05)$, whereas, no significant impact of relative humidity was found on population of $A$. soccata $(\mathrm{r}=-0.312, \mathrm{p}=>0.05)$, A. suturalis $(\mathrm{r}=-0.455, \mathrm{p}=>0.05)$ and $C$. sexmaculata $(\mathrm{r}=-0.0652, \mathrm{p}=>0.05)$. Similar to relative humidity, wind also exhibited highly significant, moderate and negative impact on population fluctuation of $R$. maidis $(\mathrm{r}=-$ 0.3586, $\mathrm{p}=<0.05), C$. mbila $(\mathrm{r}=-0.2582, \mathrm{p}$ $=<0.05), F$. occidentali $(\mathrm{r}=-0.2806, \mathrm{p}=<$ $0.05)$ and $C$. sexmaculata $(\mathrm{r}=-0.2005, \mathrm{p}=<$ $0.05)$. No significant impact was recorded between wind and A. soccata $(\mathrm{r}=-0.0731, \mathrm{p}$ $=>0.05)$, A. suturalis $(\mathrm{r}=-0.0842, \mathrm{p}=>$ $0.05)$ population.

Table 1. Weekly mean population of aphids (Rhospalosipum maidis) on various colored sticky traps.

\begin{tabular}{|c|c|c|c|c|c|c|}
\hline Date & Yellow & Blue & Green & White & Transparent & Over all mean \\
\hline $\mathbf{2 / 3 / 1 9}$ & $13.75 \pm 5.54$ & $12.00 \pm 2.16$ & $8.75 \pm 2.39$ & $10.00 \pm 2.04$ & $7.25 \pm 0.85$ & $10.35 \pm 1.15 \mathrm{f}$ \\
\hline $\mathbf{8 / 3 / 1 9}$ & $52.25 \pm 6.65$ & $36.25 \pm 5.01$ & $41.00 \pm 4.14$ & $21.75 \pm 3.75$ & $9.25 \pm 0.85$ & $32.10 \pm 7.52 \mathrm{~d}$ \\
\hline $\mathbf{1 5 / 3 / 1 9}$ & $97.50 \pm 13.73$ & $63.25 \pm 4.97$ & $52.25 \pm 4.61$ & $27.75 \pm 3.90$ & $14.00 \pm 2.16$ & $50.95 \pm 14.53 \mathrm{c}$ \\
\hline $\mathbf{2 2 / 3 / 1 9}$ & $108.75 \pm 13.61$ & $70.00 \pm 9.04$ & $51.75 \pm 6.43$ & $32.00 \pm 3.76$ & $18.75 \pm 2.10$ & $56.25 \pm 15.75 \mathrm{c}$ \\
\hline $\mathbf{3 1 / 3 / 1 9}$ & $206.25 \pm 19.67$ & $122.00 \pm 20.10$ & $106.00 \pm 13.81$ & $46.75 \pm 10.50$ & $28.50 \pm 5.44$ & $101.90 \pm 31.42 \mathrm{a}$ \\
\hline $\mathbf{6 / 4 / 1 9}$ & $131.75 \pm 18.71$ & $77.75 \pm 9.78$ & $72.75 \pm 9.59$ & $28.75 \pm 5.07$ & $16.00 \pm 2.74$ & $65.40 \pm 20.48 \mathrm{~b}$ \\
\hline $\mathbf{1 3 / 4 / 1 9}$ & $59.50 \pm 6.08$ & $40.00 \pm 5.76$ & $3.25 \pm 0.75$ & $10.50 \pm 3.69$ & $7.50 \pm 1.44$ & $24.15 \pm 10.96 \mathrm{de}$ \\
\hline $\mathbf{2 0 / 4 / 1 9}$ & $26.00 \pm 7.07$ & $5.25 \pm 2.29$ & $15.25 \pm 3.90$ & $6.00 \pm 3.19$ & $7.25 \pm 5.02$ & $11.95 \pm 3.94 \mathrm{f}$ \\
\hline $\mathbf{2 7 / 4 / 1 9}$ & $5.75 \pm 1.93$ & 0 & $2.25 \pm 1.11$ & $0.75 \pm 0.48$ & 0 & $1.75 \pm 1.08 \mathrm{~g}$ \\
\hline $\mathbf{2 / 5 / 1 9}$ & $45.25 \pm 10.61$ & $31.50 \pm 3.93$ & $36.25 \pm 5.01$ & $23.00 \pm 3.76$ & $14.50 \pm 4.17$ & $30.10 \pm 5.31 \mathrm{~d}$ \\
\hline $\mathbf{9 / 5 / 1 9}$ & $38.50 \pm 10.25$ & $13.50 \pm 3.10$ & $22.00 \pm 3.92$ & $14.50 \pm 2.90$ & $12.75 \pm 2.14$ & $20.25 \pm 4.85 \mathrm{e}$ \\
\hline $\mathbf{1 6 / 5 / 1 9}$ & $2.00 \pm 0.71$ & 0 & $0.75 \pm 0.25$ & 0 & 0 & $0.55 \pm 0.39 \mathrm{~g}$ \\
\hline $\begin{array}{c}\text { Overall } \\
\text { Mean }\end{array}$ & $56.23 \pm 16.18 \mathrm{a}$ & $33.68 \pm 10.00 \mathrm{~b}$ & $29.45 \pm 8.64 \mathrm{~b}$ & $15.84 \pm 3.89 \mathrm{c}$ & $9.70 \pm 2.25 \mathrm{~d}$ & \\
\hline
\end{tabular}

Appendix I. ANOVA for the population of $R$. maidis on different colored sticky traps

\begin{tabular}{|c|c|c|c|c|c|}
\hline Source & DF & SS & MS & F & P \\
\hline Date & 13 & 236942 & 18226.3 & 108.26 & $\mathbf{0 . 0 0 0 0}$ \\
\hline Traps & 4 & 73332 & 18333.1 & 108.90 & $\mathbf{0 . 0 0 0 0}$ \\
\hline Date * Traps & $\mathbf{5 2}$ & $\mathbf{9 5 6 4 8}$ & 1839.4 & 10.93 & $\mathbf{0 . 0 0 0 0}$ \\
\hline Error & $\mathbf{2 1 0}$ & $\mathbf{3 5 3 5 5}$ & 168.4 & & \\
\hline Total & $\mathbf{2 7 9}$ & $\mathbf{4 4 1 2 7 8}$ & & & \\
\hline
\end{tabular}


Rajput et al.

Table 2. Weekly mean population of leafhopper (Cicadulina mbila) on various colored sticky traps

\begin{tabular}{|c|c|c|c|c|c|c|}
\hline Date & Yellow & Blue & Green & White & Transparent & Overall Mean \\
\hline $\mathbf{2 / 3 / 1 9}$ & $15.25 \pm 2.14$ & $11.50 \pm 3.12$ & $13.75 \pm 6.25$ & $11.25 \pm 3.15$ & $7.00 \pm 1.22$ & $11.75 \pm 1.40 \mathrm{ef}$ \\
\hline $\mathbf{8 / 3 / 1 9}$ & $25.00 \pm 6.12$ & $16.25 \pm 5.54$ & $11.25 \pm 6.57$ & $11.25 \pm 3.15$ & $12.50 \pm 5.95$ & $\begin{array}{c}15.25 \pm 2.602 \\
\text { cdef }\end{array}$ \\
\hline $\mathbf{1 5 / 3 / 1 9}$ & $22.50 \pm 9.46$ & $17.50 \pm 1.44$ & $21.25 \pm 5.54$ & $18.75 \pm 4.27$ & $20.00 \pm 6.12$ & $20 \pm 0.88 \mathrm{cde}$ \\
\hline $\mathbf{2 2 / 3 / 1 9}$ & $31.25 \pm 8.26$ & $28.75 \pm 8.75$ & 26.25 .6 .25 & $25.00 \pm 5.00$ & $27.50 \pm 7.50$ & $23.75 \pm 3.89 \mathrm{~cd}$ \\
\hline $\mathbf{3 1 / 3 / 1 9}$ & $133.00 \pm 27.67$ & $70.75 \pm 20.30$ & $90.00 \pm 26.39$ & $52.50 \pm 18.43$ & $62.50 \pm 29.31$ & $81.75 \pm 14.22 \mathrm{~b}$ \\
\hline $\mathbf{6 / 4 / 1 9}$ & $187.50 \pm 42.70$ & $125.00 \pm 32.27$ & $106.25 \pm 41.30$ & $112.50 \pm 31.46$ & $118.75 \pm 37.33$ & $130 \pm 14.71 \mathrm{a}$ \\
\hline $\mathbf{1 3 / 4 / 1 9}$ & $46.00 \pm 19.65$ & $22.50 \pm 6.29$ & $33.75 \pm 9.87$ & $23.75 \pm 9.89$ & $25.50 \pm 8.23$ & $30.3 \pm 4.39 \mathrm{c}$ \\
\hline $\mathbf{2 0 / 4 / 1 9}$ & $21.50 \pm 3.50$ & $10.50 \pm 2.10$ & $16.25 \pm 4.25$ & $9.75 \pm 2.32$ & 6.25 .2 .02 & $12.85 \pm 2.679 \mathrm{def}$ \\
\hline $\mathbf{2 7 / 4 / 1 9}$ & $9.25 \pm 4.35$ & $3.75 \pm 1.31$ & $3.00 \pm 1.22$ & $4.50 \pm 2.22$ & $3.75 \pm 2.39$ & $4.85 \pm 1.13 \mathrm{ef}$ \\
\hline $\mathbf{2 / 5 / 1 9}$ & $22.50 \pm 7.46$ & $13.75 \pm 4.13$ & $24.25 \pm 4.25$ & $14.00 \pm 2.16$ & $12.25 \pm 3.09$ & $17.35 \pm 2.49 \mathrm{cde}$ \\
\hline $\mathbf{9 / 5 / 1 9}$ & $20.75 \pm 5.34$ & $9.00 \pm 2.74$ & $18.50 \pm 6.40$ & $6.25 \pm 2.46$ & $5.50 \pm 2.22$ & $12 \pm 3.19 \mathrm{def}$ \\
\hline $\mathbf{1 6 / 5 / 1 9}$ & $11.00 \pm 4.43$ & $2.73 \pm 1.11$ & $4.50 \pm 2.06$ & $4.75 \pm 3.30$ & $4.25 \pm 2.10$ & $5.44 \pm 1.43 \mathrm{def}$ \\
\hline $\mathbf{2 3 / 5 / 1 9}$ & $9.25 \pm 4.35$ & $3.75 \pm 1.31$ & $3.00 \pm 1.22$ & $4.50 \pm 2.22$ & $3.75 \pm 2.39$ & $4.85 \pm 1.13 \mathrm{ef}$ \\
\hline $\mathbf{3 0 / 5 / 1 9}$ & $1.75 \pm 1.18$ & $1.00 \pm 1.00$ & $0.50 \pm 0.50$ & $1.50 \pm 0.96$ & $0.75 \pm 0.75$ & $1.1 \pm 0.23 \mathrm{f}$ \\
\hline $\begin{array}{c}\text { Overall } \\
\text { Mean }\end{array}$ & $43.02 \pm 15.91 \mathrm{a}$ & $24.08 \pm 10.32 \mathrm{~b}$ & $28.95 \pm 9.55 \mathrm{~b}$ & $23.14 \pm 8.82 \mathrm{~b}$ & $24.22 \pm 9.64 \mathrm{~b}$ & \\
\hline
\end{tabular}

Appendix II. ANOVA for the population of $C$. mbila on different colored sticky traps

\begin{tabular}{|c|c|c|c|c|c|}
\hline Source & DF & SS & MS & F & P \\
\hline Date & $\mathbf{1 3}$ & $\mathbf{3 3 3 4 3 8}$ & $\mathbf{2 5 6 4 9 . 1}$ & $\mathbf{3 9 . 9 9}$ & $\mathbf{0 . 0 0 0 0}$ \\
\hline Traps & $\mathbf{4}$ & $\mathbf{1 2 6 2 6}$ & $\mathbf{3 1 5 6 . 5}$ & $\mathbf{4 . 9 2}$ & $\mathbf{0 . 0 0 0 8}$ \\
\hline Date ${ }^{*}$ Traps & $\mathbf{5 2}$ & $\mathbf{2 5 5 0 9}$ & $\mathbf{4 9 0 . 6}$ & $\mathbf{0 . 7 6}$ & $\mathbf{0 . 8 7 3 6}$ \\
\hline Error & $\mathbf{2 1 0}$ & $\mathbf{1 3 4 6 7 9}$ & $\mathbf{6 4 1 . 3}$ & & \\
\hline Total & $\mathbf{2 7 9}$ & $\mathbf{5 0 6 2 5 2}$ & & & \\
\hline
\end{tabular}

Table 3. Weekly mean population of thrips (Frankliniella occidentali) on various colored sticky traps

\begin{tabular}{|c|c|c|c|c|c|c|}
\hline Date & Yellow & Blue & Green & White & Transparent & Overall Mean \\
\hline $\mathbf{2 / 3 / 1 9}$ & $11.00 \pm 3.32$ & $6.50 \pm 1.85$ & $9.25 \pm 2.53$ & $5.00 \pm 1.22$ & $3.50 \pm 1.71$ & $7.05 \pm 1.37 \mathrm{de}$ \\
\hline $\mathbf{8 / 3 / 1 9}$ & $25.00 \pm 8.66$ & $31.25 \pm 4.27$ & $10.25 \pm 3.42$ & $20.00 \pm 7.91$ & $22.50 \pm 9.46$ & $21.8 \pm 3.44 \mathrm{~cd}$ \\
\hline $\mathbf{1 5 / 3 / 1 9}$ & $42.50 \pm 13.15$ & $18.75 \pm 5.15$ & $25.00 \pm 9.57$ & $15.00 \pm 4.56$ & $30.00 \pm 9.13$ & $26.25 \pm 4.81 \mathrm{c}$ \\
\hline $\mathbf{2 2 / 3 / 1 9}$ & $37.50 \pm 15.48$ & $22.50 \pm 9.46$ & $20.00 \pm 7.07$ & $30.00 \pm 5.77$ & $15.00 \pm 2.89$ & $25 \pm 3.95 \mathrm{c}$ \\
\hline $\mathbf{3 1 / 3 / 1 9}$ & $187.50 \pm 31.46$ & $112.50 \pm 31.46$ & $17.75 \pm 5.92$ & $175.00 \pm 48.56$ & $117.50 \pm 31.19$ & $122.05 \pm 3.06 \mathrm{a}$ \\
\hline $\mathbf{6 / 4 / 1 9}$ & $92.50 \pm 39.45$ & $75.00 \pm 27.84$ & $40.00 \pm 14.14$ & $47.50 \pm 10.31$ & $51.25 \pm 17.12$ & $61.25 \pm 9.76 \mathrm{~b}$ \\
\hline
\end{tabular}




\begin{tabular}{|c|c|c|c|c|c|c|}
\hline $\mathbf{1 3 / 4} / 19$ & $3.25 \pm 2.29$ & $0.25 \pm 0.25$ & $1.25 \pm 0.95$ & $1.00 \pm 1.00$ & $2.25 \pm 1.03$ & $1.60 \pm 0.52 \mathrm{e}$ \\
\hline $\mathbf{2 0 / 4} / 19$ & $2.50 \pm 0.87$ & $0.25 \pm 0.25$ & $0.75 \pm 0.48$ & $0.50 \pm 0.29$ & 0 & $0.8 \pm 0.44 \mathrm{e}$ \\
\hline $\mathbf{2 7 / 4 / 1 9}$ & 0 & 0 & 0 & 0 & 0 & $0.00 \pm 0.00 \mathrm{e}$ \\
\hline $\mathbf{2 / 5} / \mathbf{1 9}$ & $17.75 \pm 3.42$ & $14.00 \pm 2.94$ & $16.25 \pm 2.25$ & $13.75 \pm 3.94$ & $9.75 \pm 1.55$ & $14.3 \pm 1.36 \mathrm{cde}$ \\
\hline $\mathbf{9 / 5} / \mathbf{1 9}$ & $15.75 \pm 2.95$ & $8.00 \pm 1.41$ & $12.75 \pm 2.59$ & $6.00 \pm 1.83$ & $2.27 \pm 1.11$ & $8.95 \pm 2.40 \mathrm{de}$ \\
\hline $\mathbf{1 6 / 5} / \mathbf{1 9}$ & 0 & 0 & 0 & 0 & 0 & $0.00 \pm 0.00 \mathrm{e}$ \\
\hline $\mathbf{2 3 / 5} / 19$ & 0 & 0 & 0 & 0 & 0 & $0.00 \pm 0.00 \mathrm{e}$ \\
\hline $\mathbf{3 0 / 5} / 19$ & 0 & 0 & 0 & 0 & 0 & $0.00 \pm 0.00 \mathrm{e}$ \\
\hline $\begin{array}{c}\text { Overall } \\
\text { Mean }\end{array}$ & $31.08 \pm 13.81 \mathrm{a}$ & $20.64 \pm 8.91 \mathrm{~b}$ & $11.78 \pm 3.23 \mathrm{c}$ & $22.41 \pm 12.32 \mathrm{~b}$ & $18.14 \pm 8.63 \mathrm{bc}$ & \\
\hline
\end{tabular}

Appendix III. ANOVA for the population of $F$. occidentali on different colored sticky traps

\begin{tabular}{|c|c|c|c|c|c|}
\hline Source & DF & SS & MS & F & P \\
\hline Date & 13 & $\mathbf{2 9 6 0 7 0}$ & $\mathbf{2 2 7 7 4 . 6}$ & $\mathbf{4 2 . 1 5}$ & $\mathbf{0 . 0 0 0 0}$ \\
\hline Traps & $\mathbf{4}$ & $\mathbf{1 1 9 1 5}$ & $\mathbf{2 9 7 8 . 8}$ & $\mathbf{5 . 5 1}$ & $\mathbf{0 . 0 0 0 3}$ \\
\hline Date ${ }^{*}$ Traps & $\mathbf{5 2}$ & $\mathbf{7 2 4 0 1}$ & $\mathbf{1 3 9 2 . 3}$ & $\mathbf{2 . 5 8}$ & $\mathbf{0 . 0 0 0 0}$ \\
\hline Error & $\mathbf{2 1 0}$ & $\mathbf{1 1 3 4 6 7}$ & $\mathbf{5 4 0 . 3}$ & & \\
\hline Total & $\mathbf{2 7 9}$ & $\mathbf{4 9 3 8 5 4}$ & & & \\
\hline
\end{tabular}

Table 4. Weekly mean population of shoot fly (Atherigona soccata) population on various colored sticky traps

\begin{tabular}{|c|c|c|c|c|c|c|}
\hline Date & Yellow & Blue & Green & White & Transparent & Overall Mean \\
\hline $\mathbf{2 / 3} / \mathbf{1 9}$ & $4.75 \pm 1.25$ & $1.50 \pm 0.29$ & $2.25 \pm 0.63$ & $1.75 \pm 0.85$ & $0.50 \pm 0.29$ & $2.15 \pm 0.70 \mathrm{c}$ \\
\hline $\mathbf{8 / 3 / 1 9}$ & $5.50 \pm 1.66$ & $4.00 \pm 1.41$ & $0.75 \pm 0.48$ & $1.75 \pm 0.85$ & $1.75 \pm 1.18$ & $2.75 \pm 0.86 \mathrm{bc}$ \\
\hline $\mathbf{1 5 / 3 / 1 9}$ & $6.75 \pm 1.38$ & $1.00 \pm 0.41$ & $0.75 \pm 0.48$ & $0.50 \pm 0.29$ & $2.00 \pm 0.71$ & $2.2 \pm 1.16 \mathrm{c}$ \\
\hline $\mathbf{2 2 / 3 / 1 9}$ & $1.00 \pm 0.41$ & $0.50 \pm 0.29$ & $0.75 \pm 0.48$ & 0 & 0 & $0.45 \pm 0.2 \mathrm{~d}$ \\
\hline $\mathbf{3 1 / 3 / 1 9}$ & $7.50 \pm 4.79$ & $2.50 \pm 1.44$ & $2.00 \pm 1.08$ & $1.25 \pm 0.48$ & $2.25 \pm 1.31$ & $3.1 \pm 1.11 \mathrm{bc}$ \\
\hline $\mathbf{6 / 4 / 1 9}$ & 0 & 0 & 0 & 0 & 0 & $0.00 \pm 0.00 \mathrm{~d}$ \\
\hline $\mathbf{1 3 / 4} / 19$ & 0 & 0 & 0 & 0 & 0 & $0.00 \pm 0.00 \mathrm{~d}$ \\
\hline $\mathbf{2 0 / 4 / 1 9}$ & 0 & 0 & 0 & 0 & 0 & $0.00 \pm 0.00 \mathrm{~d}$ \\
\hline $\mathbf{2 7 / 4 / 1 9}$ & 0 & 0 & 0 & 0 & 0 & $0.00 \pm 0.00 \mathrm{~d}$ \\
\hline $\mathbf{2 / 5 / 1 9}$ & $12.50 \pm 2.78$ & $6.25 \pm 2.02$ & $8.25 \pm 2.32$ & $3.75 \pm 1.55$ & $2.50 \pm 1.55$ & $6.65 \pm 1.76 \mathrm{a}$ \\
\hline $\mathbf{9 / 5 / 1 9}$ & $9.00 \pm 2.08$ & $2.00 \pm 0.85$ & $4.25 \pm 1.44$ & $1.50 \pm 0.87$ & $2.00 \pm 0.71$ & $3.75 \pm 1.39 \mathrm{~b}$ \\
\hline $\mathbf{1 6 / 5 / 1 9}$ & 0 & 0 & 0 & 0 & 0 & $0.00 \pm 0.00 \mathrm{~d}$ \\
\hline $\mathbf{2 3 / 5 / 1 9}$ & 0 & 0 & 0 & 0 & 0 & $0.00 \pm 0.00 \mathrm{~d}$ \\
\hline $\mathbf{3 0 / 5 / 1 9}$ & 0 & 0 & 0 & 0 & 0 & $0.00 \pm 0.00 \mathrm{~d}$ \\
\hline
\end{tabular}




\begin{tabular}{|c|c|c|c|c|c|c|}
\hline $\begin{array}{c}\text { Overall } \\
\text { Mean }\end{array}$ & $3.35 \pm 1.09 \mathrm{a}$ & $1.26 \pm 0.48 \mathrm{~b}$ & $1.35 \pm 0.60 \mathrm{~b}$ & $0.75 \pm 0.28 \mathrm{~b}$ & $0.78 \pm 0.26 \mathrm{~b}$ & \\
\hline
\end{tabular}

Appendix IV. ANOVA for the population of A. soccata on different colored sticky traps

\begin{tabular}{|c|c|c|c|c|c|}
\hline Source & DF & SS & MS & F & P \\
\hline Date & 13 & $\mathbf{1 0 6 9 . 4 5}$ & $\mathbf{8 2 . 2 6 5 1}$ & $\mathbf{2 0 . 0 6}$ & $\mathbf{0 . 0 0 0 0}$ \\
\hline Traps & 4 & 257.37 & $\mathbf{6 4 . 3 4 2 9}$ & $\mathbf{1 5 . 6 9}$ & $\mathbf{0 . 0 0 0 0}$ \\
\hline Date ${ }^{*}$ Traps & $\mathbf{5 2}$ & $\mathbf{4 6 1 . 9 3}$ & $\mathbf{8 . 8 8 3 2}$ & $\mathbf{2 . 1 7}$ & $\mathbf{0 . 0 0 0 1}$ \\
\hline Error & $\mathbf{2 1 0}$ & $\mathbf{8 6 1 . 2 5}$ & $\mathbf{4 . 1 0 1 2}$ & & \\
\hline Total & $\mathbf{2 7 9}$ & $\mathbf{2 6 5 0 . 0 0}$ & & & \\
\hline
\end{tabular}

Table 5. Weekly mean population of mirid bugs (Adelphocoris suturalis) on various colored sticky traps

\begin{tabular}{|c|c|c|c|c|c|c|}
\hline Date & Yellow & Blue & Green & White & Transparent & Overall Mean \\
\hline $\mathbf{2 / 3 / 1 9}$ & $0.75 \pm 0.48$ & $0.25 \pm 0.25$ & $0.50 \pm 0.29$ & $0.25 \pm 0.25$ & 0 & $0.35 \pm 0.13 \mathrm{cde}$ \\
\hline $\mathbf{8 / 3 / 1 9}$ & $1.50 \pm 1.96$ & $0.74 \pm 0.48$ & $0.25 \pm 0.25$ & $1.25 \pm 0.95$ & $1.00 \pm 0.46$ & $0.95 \pm 0.22 \mathrm{ab}$ \\
\hline $\mathbf{1 5 / 3 / 1 9}$ & $0.75 \pm 0.48$ & 0 & $0.50 \pm 0.29$ & 0 & 0 & $0.25 \pm 0.16 \mathrm{de}$ \\
\hline $\mathbf{2 2 / 3 / 1 9}$ & 0 & 0 & 0 & 0 & 0 & $0.00 \pm 0.00 \mathrm{e}$ \\
\hline $\mathbf{3 1 / 3 / 1 9}$ & $1.50 \pm 0.29$ & $1.25 \pm 0.63$ & $0.50 \pm 0.29$ & $2.25 \pm 0.63$ & $1.25 \pm 0.63$ & $1.35 \pm 0.28 \mathrm{a}$ \\
\hline $\mathbf{6 / 4 / 1 9}$ & $1.50 \pm 0.65$ & $0.75 \pm 0.48$ & $1.00 \pm 0.71$ & $0.50 \pm 0.50$ & $0.75 \pm 0.48$ & $0.90 \pm 0.17 \mathrm{ab}$ \\
\hline $\mathbf{1 3 / 4 / 1 9}$ & $1.25 \pm 0.48$ & $0.75 \pm 0.48$ & $1.00 \pm 0.41$ & $0.75 \pm 0.48$ & $0.25 \pm 0.25$ & $0.80 \pm 0.17 \mathrm{bc}$ \\
\hline $\mathbf{2 0 / 4 / 1 9}$ & $1.00 \pm 0.41$ & 0 & $0.75 \pm 0.48$ & 0 & 0 & $0.35 \pm 0.22 \mathrm{cde}$ \\
\hline $\mathbf{2 7 / 4 / 1 9}$ & $0.75 \pm 0.48$ & 0 & $0.25 \pm 0.25$ & 0 & $0.50 \pm 0.29$ & $0.30 \pm 0.15 \mathrm{de}$ \\
\hline $\mathbf{2 / 5 / 1 9}$ & $1.25 \pm 0.48$ & $0.50 \pm 0.29$ & $1.00 \pm 0.41$ & $0.50 \pm 0.29$ & $0.25 \pm 0.25$ & $0.70 \pm 0.18 \mathrm{bcd}$ \\
\hline $\mathbf{9 / 5 / 1 9}$ & $2.50 \pm 0.87$ & 0 & $1.50 \pm 0.87$ & 0 & 0 & $0.80 \pm 0.51 \mathrm{bc}$ \\
\hline $\mathbf{1 6 / 5 / 1 9}$ & 0 & 0 & 0 & 0 & 0 & $0.00 \pm 0.00 \mathrm{e}$ \\
\hline $\mathbf{2 3 / 5 / 1 9}$ & $0.75 \pm 0.48$ & 0 & $0.25 \pm 0.25$ & 0 & $0.50 \pm 0.29$ & $0.30 \pm 0.15 \mathrm{de}$ \\
\hline $\mathbf{3 0 / 5 / 1 9}$ & 0 & 0 & 0 & 0 & 0 & $0.00 \pm 0.00 \mathrm{e}$ \\
\hline $\begin{array}{c}\text { Overall } \\
\text { Mean }\end{array}$ & $1.06 \pm 0.18 \mathrm{a}$ & $0.33 \pm 0.11 \mathrm{~b}$ & $0.60 \pm 0.12 \mathrm{~b}$ & $0.42 \pm 0.1 \mathrm{~b}$ & $0.33 \pm 0.12 \mathrm{~b}$ & \\
\hline
\end{tabular}

Appendix V. ANOVA for the population of A. suturalis on different colored sticky traps

\begin{tabular}{|c|c|c|c|c|c|}
\hline Source & DF & SS & MS & F & P \\
\hline Date & 13 & 42.243 & $\mathbf{3 . 2 4 9 4 5}$ & $\mathbf{5 . 4 6}$ & $\mathbf{0 . 0 0 0 0}$ \\
\hline Traps & 4 & 19.621 & $\mathbf{4 . 9 0 5 3 6}$ & $\mathbf{8 . 2 4}$ & $\mathbf{0 . 0 0 0 0}$ \\
\hline Date * Traps & $\mathbf{5 2}$ & $\mathbf{3 1 . 0 7 9}$ & $\mathbf{0 . 5 9 7 6 6}$ & $\mathbf{1 . 0 0}$ & $\mathbf{0 . 4 7 5 0}$ \\
\hline Error & $\mathbf{2 1 0}$ & $\mathbf{1 2 5 . 0 0 0}$ & $\mathbf{0 . 5 9 5 2 4}$ & & \\
\hline Total & $\mathbf{2 7 9}$ & $\mathbf{2 1 7 . 9 4 3}$ & & & \\
\hline
\end{tabular}


Table 6. Weekly mean population of zigzag beetle (Cheilomenes sexmaculata) on various colored sticky traps

\begin{tabular}{|c|c|c|c|c|c|c|}
\hline Date & Yellow & Blue & Green & White & Transparent & Overall Mean \\
\hline 2/3/19 & $1.50 \pm 0.65$ & $0.50 \pm 0.29$ & $1.75 \pm 0.85$ & $0.75 \pm 0.48$ & $0.50 \pm 0.29$ & $1.00 \pm 0.26 \mathrm{bcd}$ \\
\hline $8 / 3 / 19$ & $2.50 \pm 0.96$ & $1.50 \pm 0.96$ & $1.25 \pm 0.95$ & $0.75 \pm 0.48$ & $0.50 \pm 0.29$ & $1.30 \pm 0.35 \mathrm{abc}$ \\
\hline $15 / 3 / 19$ & $2.00 \pm 0.71$ & $1.00 \pm 0.41$ & $0.75 \pm 0.48$ & $0.50 \pm 0.50$ & $1.25 \pm 0.95$ & $1.10 \pm 0.26 \mathrm{abcd}$ \\
\hline $22 / 3 / 19$ & $1.50 \pm 0.96$ & $0.75 \pm 0.48$ & $0.75 \pm 0.48$ & $1.00 \pm 0.50$ & $0.25 \pm 0.25$ & $0.85 \pm 0.20 \mathrm{~cd}$ \\
\hline $31 / 3 / 19$ & $2.50 \pm 1.26$ & $1.76 \pm 0.48$ & $1.50 \pm 0.50$ & $1.00 \pm 0.71$ & $1.50 \pm 0.65$ & $1.65 \pm 0.25 \mathrm{ab}$ \\
\hline $6 / 4 / 19$ & $0.75 \pm 0.95$ & $1.75 \pm 1.18$ & $1.50 \pm 0.96$ & $1.25 \pm 0.75$ & $1.75 \pm 0.63$ & $1.40 \pm 0.19 \mathrm{a}$ \\
\hline $13 / 4 / 19$ & $1.75 \pm 1.03$ & $1.00 \pm 0.58$ & $1.25 \pm 0.95$ & $0.25 \pm 0.25$ & $1.00 \pm 0.41$ & $1.05 \pm 0.24 \mathrm{bcd}$ \\
\hline $20 / 4 / 19$ & $1.00 \pm 0.41$ & $0.25 \pm 0.25$ & $0.75 \pm 0.48$ & 0 & 0 & $0.40 \pm 0.20 \mathrm{de}$ \\
\hline $27 / 4 / 19$ & $1.00 \pm 0.71$ & $0.75 \pm 0.48$ & $0.50 \pm 0.29$ & $0.25 \pm 0.25$ & $0.25 \pm 0.25$ & $0.55 \pm 0.15 \mathrm{de}$ \\
\hline $2 / 5 / 19$ & $3.50 \pm 0.65$ & $1.00 \pm 0.58$ & $2.00 \pm 0.71$ & $0.50 \pm 0.29$ & $0.25 \pm 0.25$ & $1.45 \pm 0.59 \mathrm{abc}$ \\
\hline $9 / 5 / 19$ & $2.00 \pm 0.71$ & $0.50 \pm 0.29$ & $1.00 \pm 0.58$ & $0.50 \pm 0.29$ & $0.25 \pm 0.25$ & $0.85 \pm 0.31 \mathrm{~cd}$ \\
\hline $16 / 5 / 19$ & 0 & 0 & 0 & 0 & 0 & $0.00 \pm 0.00 \mathrm{e}$ \\
\hline $23 / 5 / 19$ & $1.00 \pm 0.71$ & $0.75 \pm 0.48$ & $0.50 \pm 0.29$ & $0.25 \pm 0.25$ & $0.25 \pm 0.25$ & $0.55 \pm 0.15 \mathrm{de}$ \\
\hline $30 / 5 / 19$ & 0 & 0 & 0 & 0 & 0 & $0.00 \pm 0.00 \mathrm{e}$ \\
\hline $\begin{array}{c}\text { Overall } \\
\text { Mean }\end{array}$ & $1.5 \pm 0.26 \mathrm{a}$ & $0.82 \pm 0.15 b c$ & $0.94 \pm 0.16 \mathrm{~b}$ & $0.50 \pm 0.10 \mathrm{c}$ & $0.55 \pm 0.15 b c$ & \\
\hline
\end{tabular}

Appendix VI. ANOVA for the population of $C$. sexmaculata on different colored sticky traps

\begin{tabular}{|c|c|c|c|c|c|}
\hline Source & DF & SS & MS & F & P \\
\hline Date & $\mathbf{1 3}$ & $\mathbf{8 0 . 5 4 4}$ & $\mathbf{6 . 1 9 5 7}$ & $\mathbf{4 . 7 6}$ & $\mathbf{0 . 0 0 0 0}$ \\
\hline Traps & $\mathbf{4}$ & $\mathbf{4 6 . 9 5 2}$ & $\mathbf{1 1 . 7 3 7 9}$ & $\mathbf{9 . 0 1}$ & $\mathbf{0 . 0 0 0 0}$ \\
\hline Date $*$ Traps & $\mathbf{5 2}$ & $\mathbf{3 3 . 7 2 4}$ & $\mathbf{0 . 6 4 8 5}$ & $\mathbf{0 . 5 0}$ & $\mathbf{0 . 9 9 8 2}$ \\
\hline Error & $\mathbf{2 0 9}$ & $\mathbf{2 7 2 . 2 5 0}$ & $\mathbf{1 . 3 0 2 6}$ & & \\
\hline Total & $\mathbf{2 7 8}$ & & & & \\
\hline
\end{tabular}

Table 7. Pearson's correlation to determine role of abiotic factors on the performance of colored sticky traps

\begin{tabular}{|c|c|c|c|c|c|c|}
\hline Insects & $\boldsymbol{R}$. maidis & $\boldsymbol{C}$. mbila & $\boldsymbol{F}$. occidentali & A. soccata & A. suturalis & $\begin{array}{c}\boldsymbol{C} \text {. } \\
\text { sexmaculata }\end{array}$ \\
\hline \multirow{2}{*}{ Temperature } & $\mathrm{r}=0.1314$ & $\mathrm{r}=0.1921$ & $\mathrm{r}=0.1287$ & $\mathrm{r}=0.0420$ & $\mathrm{r}=0.1003$ & $\mathrm{r}=0.1207$ \\
& $\mathrm{P}=0.0282$ & $\mathrm{P}=0.0013$ & $\mathrm{P}=0.0316$ & $\mathrm{P}=0.4846$ & $\mathrm{P}=0.945$ & $\mathrm{P}=0.0440$ \\
\hline Relative & $\mathrm{r}=-0.3061$ & $\mathrm{r}=-0.1572$ & $\mathrm{r}=-0.2572$ & $\mathrm{r}=-0.312$ & $\mathrm{r}=-0.455$ & $\mathrm{r}=-0.0652$ \\
humidity & $\mathrm{P}=0.0000$ & $\mathrm{P}=0.0085$ & $\mathrm{P}=0.0000$ & $\mathrm{P}=0.6040$ & $\mathrm{P}=0.4492$ & $\mathrm{P}=0.2775$ \\
\hline \multirow{2}{*}{ Wind } & $\mathrm{r}=-0.3586$ & $\mathrm{r}=-0.2582$ & $\mathrm{r}=-0.2806$ & $\mathrm{r}=-0.0731$ & $\mathrm{r}=-0.0842$ & $\mathrm{r}=-0.2005$ \\
& $\mathrm{P}=0.0000$ & $\mathrm{P}=0.0000$ & $\mathrm{P}=0.0000$ & $\mathrm{P}=0.2237$ & $\mathrm{P}=0.1609$ & $\mathrm{P}=0.0008$ \\
\hline
\end{tabular}




\section{Discussion}

In present study, examined the relative attractiveness of maize insects toward the different colored sticky traps in field conditions. Five different colored sticky traps (white, yellow, green, blue and transparent) were randomly installed at one feet above the crop height. Thus, height of the traps was adjusted according to the crop stages. Correlation of sticky traps and abiotic factors (temperature, wind velocity and relative humidity) on insects of maize was investigated. The data was taken from $2^{\text {nd }}$ March, 2019 to $30^{\text {nd }}$ May, 2019. Six different insect species i.e., $R$. maidis, $C$. mbila, $F$. occidentali, $A$. soccata, $A$. suturalis and $C$. sexmaculata were responded to various colored traps in maize field. The collected data indicated that the all insects were strongly attracted on yellow sticky traps as compared to blue, green, white and transparent.

The results of this study are in accordance with many previously research experiment. [19] reported that among several installed sticky colored cards, the adults of predators were highly attracted towards the yellow sticky cards, especially before pollen shed. Blue colored traps captured more bumble bees (Bombus spp.) [20] whereas sevenspotted ladybird, Coccinella septempunctata (L.) were more attracted on yellow colored trap in alfalfa [21] Studied on the population dynamics of maize insect pests and their associated natural enemies in maize field with three sampling methods to count the predator's population showed that the highest numbers of thrips were attracted to yellow adhesive card and dark colored cards in circular shape than on blue, or green traps $[22,23]$. However red, green, and yellow colored cards were more effective to attract Elatobium. abietinum than blue, white, and dark adhesive cards [19]. Moreover, [24] Found that yellow adhesive cards were more effected to attract adult mango hoppers, while pink and purple colors were found less attractive. The use of sticky traps gives a basic technique for monitoring relative evaluations of pest population with small efforts mostly used in integrated pest management programs in diverse agricultural crops [13]. Similar observations on yellow, white, or blue adhesive cards are presently suggested for detecting and managing thrips [25].

The study results also found that weather parameters i.e., temperature, relative humidity and wind velocity showed a weak and significant impact on the population of R. maidis, F. occidentali, C. mbila, and $C$. sexmaculata, whereas, they did not show any important role in the population of $A$. soccata and $A$. suturalis. Many previous studies also confirmed the role of temperature, relative humidity and wind in the population regulation of many important pests of crops. it has been reported that the insects are highly susceptible to the environmental conditions [5] revealed the influence of abiotic stresses on the incidence of maize insect pests on different maize varieties. The highest infestation was observed at low relative humidity high and optimum temperature ranges. Furthermore, sunshine and wind velocity were positively correlated with pest population.

\section{Conclusion and Recommendations}

From the present experiment, it has been concluded that yellow sticky traps showed a strong attractiveness for $R$. maidis, C. mbila, $F$. occidentali, A. soccata, A. suturalis and $C$. sexmaculata. Whereas, according to overall weekly observation the maximum numbers of insects were scouted by colored traps during March and April in maize corp. Furthermore, a-biotic factors (temperature, relative humidity, wind velocity) significantly affected on the performance of colored sticky traps to attract various insects. Based on the experiment following suggestions are recommended. Yellow, 
sticky traps should be used for the early detection and monitoring the populations of different insects on maize crop.

\section{Authors' contributions}

Conceived and designed the experiments: A Rajput, Performed the experiments: A Rajput, JA Soomro, AL Baloch \& GQ Junejo, Analyzed the data: A Rajput, Contributed materials/ analysis/ tools: A Rajput \& JA Soomro, Wrote the paper: A Rajput.

\section{References}

1. Khaliq $\mathrm{T}$, Mahmood $\mathrm{T} \&$ Masood $\mathrm{A}$ (2004). Effectiveness of farmyard manure, poultry manure and nitrogen for corn (Zea mays) productivity. Inter $J$ of Agric and Biol 2: 260-263.

2. CIMYYT (2019). Why Maize. Retrieved July 29, 2019, from https://maize.org/why-maize/

3. Acharya V \& Young B (2008). A review of the potential of bio-ethanol in New Zealand. Bull of Sci, Technol \& Soc 28(2): pp 143-148.

4. Tariq M \& Iqbal H (2010). Maize in Pakistan: an overview. Kasetsart J (Nat Sci) 44(5): 757.

5. Zulfiqar U, Jurivich DA, Gao W \& Singer DH (2010). Relation of high heart rate variability to healthy longevity. American $J$ of Cardiol pp. 1181-1185.

6. Gebremdein, M. B (2018). Varietal screening for resistance against field and storage crop pests: An implication for Ethiopian crop variety development. $J$ of Plant Breeding and Crop Sci 10: 203-209.

7. Shah STH, Zamir MSI, Waseem M, Ali A, Tahir M \& Khalid WB (2009). Growth and yield response of maize (Zea mays L.) to organic and inorganic sources of nitrogen. Pak J Life Soc Sci 7(2): 108-111.

8. Demissie, G, Tefera $\mathrm{T} \&$ Tadesse A (2008). Efficacy of Silicosec, filter cake and wood ash against the maize weevil, Sitophilus zeamais Motschulsky (Coleoptera: Curculionidae) on three maize genotypes. J of Stored Prod Res 44(3): 227-231.

9. Mahmood I, SR, Imadi K, Shazadi A, Gul \& Hakeem KR (2019). Effects of pesticides on environment. (C) Springer International Publishing Switzerland K. R. Hakeem et al. (Eds.), Plant, Soil and Microbes pp. 253-266.

10. Abebe T, Melmaiee K, Berg V \& Wise RP (2010). Drought response in the spikes of barley: gene expression in the lemma, palea, awn, and seed. Funct \& Integrative Geno 10(2): 191-205.

11. Mashwani MA, Ullah F, Ahmad S, Sohail K, Shah SF \& Usman M (2015). Infestation of maize stem borer, Chilo partellus (Swinhoe) in maize stubbles and stalks. J Biol Environ Sci 7:180185.

12. Gillespie DR \& Vernon RS (1990). Trap catch of western flower thrips (Thysanoptera: Thripidae) as affected by color and height of sticky traps in mature greenhouse cucumber crops. $J$ of Eco Entomol 83(3): 971-975.

13. Gencsoylu (2018). Seasonal abundance; damage; and comparison of different heights, orientations, and directions of yellow sticky traps for sampling of Liriomyza trifolii (Diptera: Agromyzidae) in Cotton. The Great Lakes Entomol 39(1 \& 2): 4.

14. Allsopp, E (2010). Investigation into the apparent failure of chemical control for management of western flower thrips, Frankliniella occidentalis (Pergande), on plums in the Western Cape Province of South Africa. Crop Prot 29: 824-831.

15. Vinson ST, \& Scarborough (1991). Interactions

between Solenopsis invicta (Hymenoptera, Formicidae), Rhopalosiphum maidis (Homoptera, 
Aphididae), and the parasitoid Lysiphlebus testaceipes Cresson (Hymenoptera, Aphidiidae). Annals of the Entomol Soc of America 84(2): 158164.

16. Mukhtar A, Ahmed MJ, Shama A \& Mishra RK (2001). First record of coccinellid beetles (Coleoptera: Coccinellidae) on poplar, populous deltoids from North India. Indian Forest 127(8): 891-897.

17. Matteson N, Terry I, Ascoli-Christensen A \& Gilbert C (1992). Spectral efficiency of the western flower thrips, Frankliniella occidentalis. $J$ of Insect Physiol 38: 453-459.

18. Jotwani MG \& Srivastava KP (1969). Studies on sorghum lines resistant against shoot-fly, Atherigona varia, soccata Rond. Indian J of Entomol 32: $1-3$.

19. Wallis DR \& Shaw PW (2008). Evaluation of colored sticky traps for monitoring beneficial insects in apple orchards. New Zealand Plant Prot 61: 328-332.

20. Clare GD, Sucking M, Bradley SJ, Walker JTS, Shaw PW, Daly JM, McLaren GF \& Wearing CH (2000). Pheromone trap color determines catch of non-target insects. New Zealand Plant Prot 53: 216-220.
21. Hoddle MS, Robinson L \& Morgan D (2002). Attraction of thrips (Thysanoptera: Thripidae and Aeolothripidae) to colored sticky cards in a California avocado orchard. Crop Prot 21: 383-388.

22. Mainali BP \& Lim UT (2010). Circular yellow sticky trap with black background enhances attraction of Frankliniella occidentalis (Pergande) (Thysanoptera: Thripidae). Appl Entomol and Zool 45: 207-213.

23. Straw NA, Williams DT \& Green G (2011). Influence of sticky trap color and height above ground on capture of alate Elatobium abietinum (Hemiptera: Aphididae) in Sitka spruce plantations. Environ Entomol 40: 120125.

24. Saeed S, Amin MA, Saeed Q \& Farooq M (2013). Attraction of Idioscopus clypealis (Leith) (Cicadellidae: Homoptera) to sticky colored traps in mango orchard. American $J$ of Plant Sci 4: 2275-2280.

25. Tang LD, Zhao HY, Fu BL, Han Y, Liu $\mathrm{K} \& \mathrm{Wu}$ JH (2016). Colored sticky traps

to selectively survey thrips in cowpea ecos ystem. Neotropical Entomol 45: 96-101. 